\title{
9. METAMORPHIC PETROLOGY OF ODP LEG 109, HOLE 670A SERPENTINIZED PERIDOTITES: SERPENTINIZATION PROCESSES AT A SLOW SPREADING RIDGE ENVIRONMENT ${ }^{1}$
}

\author{
R. Hébert, ${ }^{2}$ A. C. Adamson, ${ }^{3}$ and S. C. Komor ${ }^{4}$
}

\begin{abstract}
In this paper we describe textural relationships in hydrated upper mantle peridotites emplaced at a nonconstructive ridge segment. Development of serpentinites and partially serpentinized peridotites takes place in four main stages: (1) pervasive serpentinization forming mainly lizardite, (2) a tensional stage forming chrysotile + talc + chlorite, (3) a deformational stage forming antigorite + tremolite, and (4) a late local tensional stage forming another generation of chrysotile veinlets. Mineral chemistry of serpentine pseudomorphs reflects in part primary mineral compositions. Olivine pseudomorphs are typically nickeliferous and depleted in aluminum and chromium. Orthopyroxene pseudomorphs have lower nickel contents and relatively high iron, aluminum, and chromium contents. Clinopyroxene pseudomorphs have very low nickel contents and relatively high aluminum and chromium contents. These chemical patterns in the serpentinites can be used to help discriminate between harzburgitic and lherzolitic protoliths. Oxygen isotopes and mineral parageneses suggest serpentine is derived from circulation of hydrothermal $\left(200^{\circ} \mathrm{C}\right)$ fluids through the peridotite body. Crystallization of tremolite, talc, and chlorite may have occurred at temperatures up to $525^{\circ} \mathrm{C}$ if $\mathrm{CO}_{2} / \mathrm{H}_{2} \mathrm{O}$ ratios were less than 0.25 . Open fissures developing in aging upper mantle provide paths for important seawater circulation through a thin basaltic carapace down to shallow mantle rocks.
\end{abstract}

\section{INTRODUCTION}

Various types of serpentinites and partly serpentinized harzburgites were recovered during Leg 109 at Site 670, located on the west flank of the Mid-Atlantic Ridge rift valley (Fig. 1). These rocks record several stages of hydration and deformation that have generated complex mineral replacement and crosscutting relationships. The aim of this paper is to understand the processes of serpentinization and the conditions of formation of these disequilibrium mineral assemblages. Detailed petrographic and petrologic analysis of these rocks reveals how replacement occurred and the types and sequences of replacement. The mineral assemblages and oxygen isotope geochemistry help to constrain the thermobarometric conditions and fluid properties that controlled the replacement processes.

\section{SUMMARY OF SERPENTINIZATION STRUCTURES AND TEXTURES}

Studies of primary mineralogy show that the initial composition of the rocks from Hole $670 \mathrm{~A}$ is bracketed between dunite and clinopyroxene-bearing harzburgite (see Komor et al., this volume and Hébert et al., this volume). These rocks exhibit various stages of serpentinization. The intensity of serpentinization, proceeding in several steps, varies from $40 \%$ to $100 \%$. Serpentinites are found in Cores $670 \mathrm{~A}-5 \mathrm{R},-7 \mathrm{R}$, and $-9 \mathrm{R}$ (Fig. 2), where 5 to 10 modal $\%$ of relict primary orthopyroxene porphyroclasts and spinel are still present. These samples represent intensively hydrated peridotites (dunites). Extensive fluid migration first produced pervasive serpentinization (lizardite phase; Aumento, 1970) and resulted

\footnotetext{
I Detrick, R., Honnorez, J., Bryan, W. B., Juteau, T., et al., 1990. Proc. $O D P$, Sci. Results, 106/109: College Station, TX (Ocean Drilling Program).

${ }^{2}$ Département de Géologie, Université Laval, Ste-Foy, Québec, Canada, GIK 7 P4.

${ }^{3}$ Ocean Drilling Program, Texas A \& M University, 1000 Discovery Drive, College Station, Texas 77843-3469.

${ }^{4}$ U.S. Geological Survey, 821 E. Interstate Avenue, Bismarck, ND 58501.
}

in the formation of mega-mesh texture, allowing further circulation of seawater and rock-seawater interaction along multiple fronts. Intergrowths and interlayering of talc, lizardite, and small amounts of antigorite provide evidence for local variability of fluid compositions and activities of $\mathrm{SiO}_{2}, \mathrm{MgO}$, and other components (see Shipboard Scientific Party, 1988). These textures are especially well developed in Samples $670 \mathrm{~A}-5 \mathrm{R}-1,45-57 \mathrm{~cm}, 670 \mathrm{~A}-5 \mathrm{R}-1,8-11 \mathrm{~cm}$, and $670 \mathrm{~A}-5 \mathrm{R}-2$, 11-17 cm. In these samples, mega-mesh textures are highlighted by late-stage or vein-filling antigorite(?) and chrysotile (identifications based on textural relationships). In Core 670A$7 R$ (Pieces 7A-7F) the serpentinites are sheared and more severely metamorphosed. A cataclastic texture is present comprising a complex mixture of lizardite replaced by talc, antigorite, and chrysotile. Overgrowths of tremolite, chlorite (clinochlore), and carbonate superimposed on this complex assemblages are observed. Centimeter-thick veins of massive serpentinite define fault planes in the cataclasized zone. This deformation is likely to be related to volume increases and the stresses resulting from serpentinization (Janecky and Seyfried, 1986).

Detailed petrography reveals that serpentinization proceeded in four main stages:

Stage 1: Pervasive serpentinization (e.g., Samples 670A$5 \mathrm{R}-1,50-52 \mathrm{~cm}$, and $670 \mathrm{~A}-9 \mathrm{R}-1,10-12 \mathrm{~cm}$ )

This stage is seen in various states of advancement. The general order of replacement is olivine, orthopyroxene, clinopyroxene, and spinel. Pale green to reddish and dark brown (weathered?) lizardite replaces olivine and forms polygonal mesh texture with structureless serpentine cores (Wicks and Whittaker, 1977; Plate 1, Fig. 1). Orthopyroxene and clinopyroxene are pseudomorphed by lizardite (bastite). Magnetite seams (0.1-0.3 $\mathrm{mm}$ wide) are observed along the primary grain boundaries and/or cleavages. The outermost rims of the pseudomorphs are replaced by fibrous chrysotile and/or flaky talc. The olivine pseudomorphs are in turn cut by chrysotile veinlets, and magnetite lines the contacts between the veinlets and unaltered olivine. The bastite pseudomorphs are partially replaced by talc at the rims or along preserved 001 cleavage 


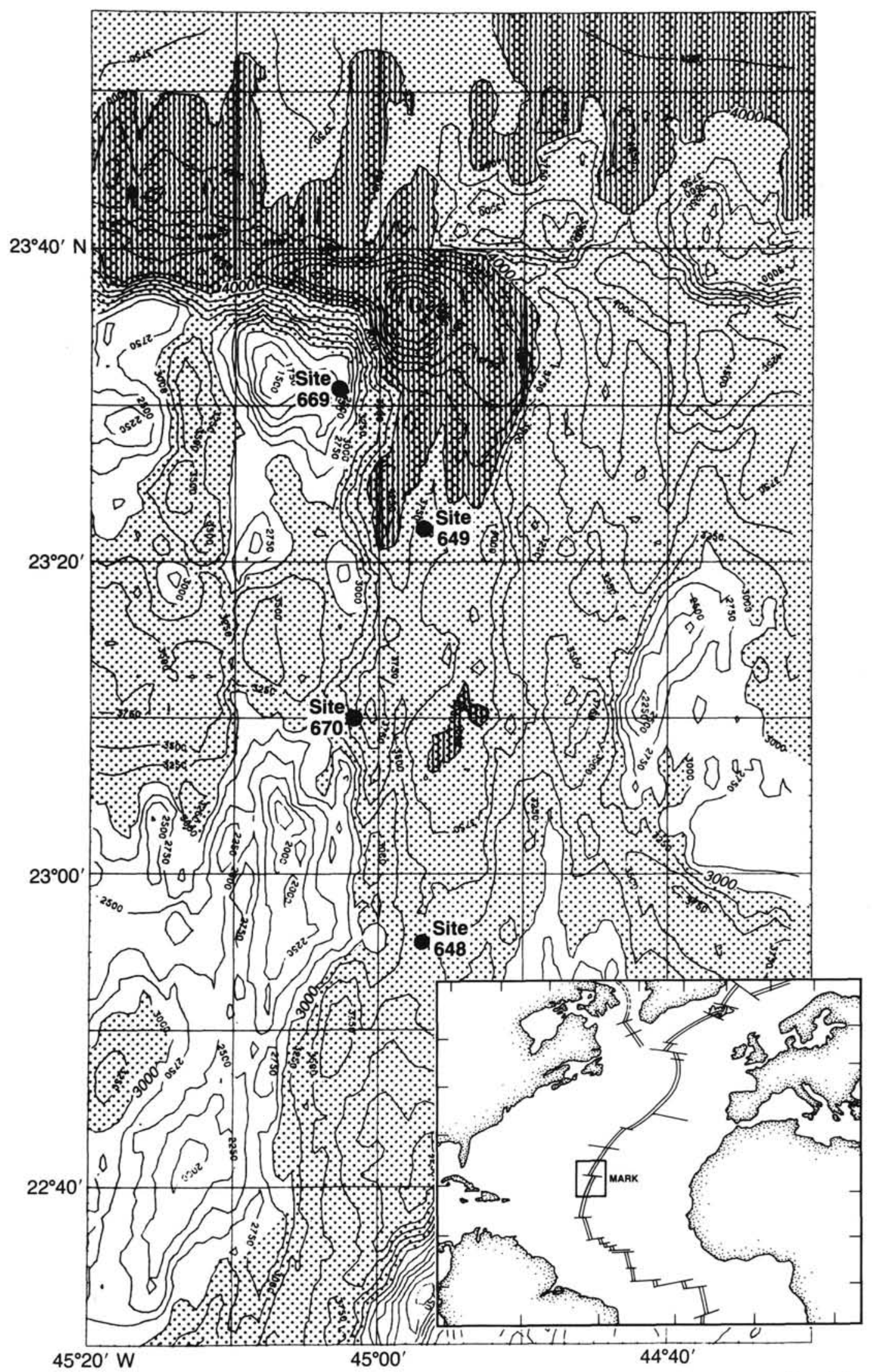

Figure 1. Sea Beam bathymetric map of the MARK area showing location of Site 670 on the west side of the Mid-Atlantic Ridge rift valley. Other ODP sites in this area are shown for reference. Contour interval $250 \mathrm{~m}$. Depths shallower than $3000 \mathrm{~m}$ are unshaded, depths between 3000 and $4000 \mathrm{~m}$ are indicated by a light stipple pattern, and depths greater than $4000 \mathrm{~m}$ are darkly shaded. After Detrick et al. (1988). 


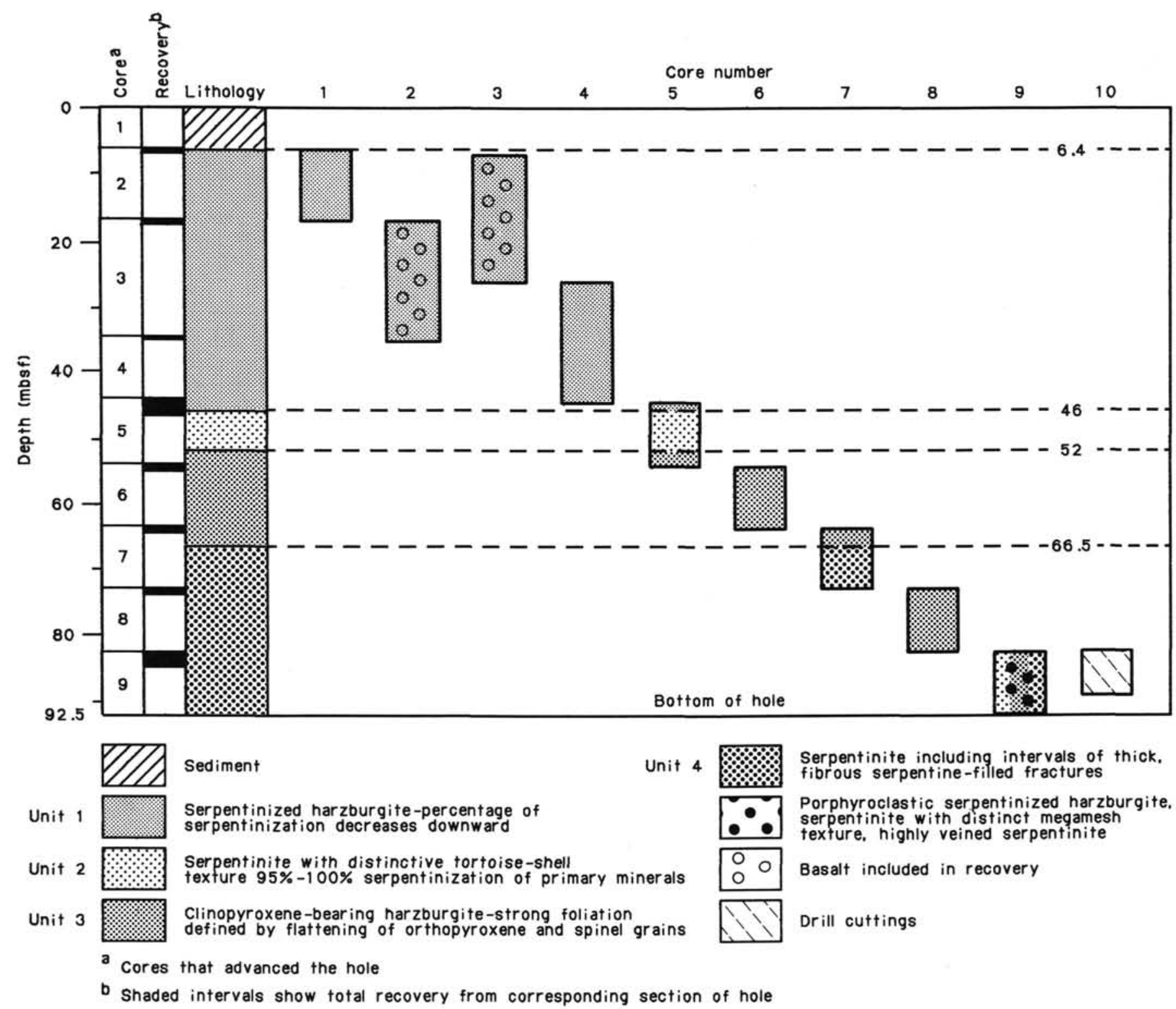

Figure 2. Synthesis of recovered cores of serpentinized rocks from Hole 670A. From Shipboard Scientific Party (1988).

planes. Translucent spinels are partially altered to opaque magnetite or ferrite-chromite around the margins and along cracks leaving fresh cores. In Sample 670A-5R-1, 111-113 cm, spinel replacement is complete. Spinels are also rimmed by chlorite with anomalous blue interference colors (e.g., Sample 670A-7R-1, 43-45 cm),

Stage 2: Tensional stage serpentinization (e.g., Sample $670 \mathrm{~A}-3 \mathrm{~W}-1,12-14 \mathrm{~cm}$ )

In this stage, chrysotile crystallizes in dilation fractures that form an orthogonal or irregular net. Chrysotile fibers grow perpendicularly to fissure walls suggesting simultaneous dilation and growth (Riordon, 1955). Magnetite granules are concentrated at adjacent fissure wall-rock contacts. In Sample 670A-7R-1, 43-45 cm, talc and chlorite accompany chrysotile growth or slightly postdate its development. This relationship is observed when the rock is deformed. These deformed areas (up to $3 \mathrm{~mm}$ across) are then invaded by talc and chlorite intergrowths or veinlets. The areas are in turn cut by chrysotile and chlorite veinlets, or form the starting point of radiating chrysotile-chlorite veinlets. The shearing sub-stage of the main tensional stage leads to the formation of sheared chrysotile veins. Chrysotile fibers in those veins form acute angles with the vein walls and magnetite veins (Plate 1, Fig. 2). This could represent an intermediate stage toward antigorite formation (Stage 3). In Sample 670A-9R-1, 10-12 cm, talc + chlorite assemblages cut the polygonal olivine pseudomorphs instead of chrysotile. This event can be explained by having heterogeneous metamorphic fluids. Stage 2 serpentinization is a process capable of generating localized high strains and stresses, with episodic cracking, that allows seawater to invade peridotite bodies (Macdonald and Fyfe, 1985).

Stage 3: Deformational stage and formation of antigorite

Local formation of antigorite (less than 5\% in mode) is accompanied by shearing oblique to the primary foliation. The shear zones are irregular $(0.5-1 \mathrm{~cm}$ thick), and are not present in all the samples studied (e.g., Sample 670A-5R-1, 111-113 $\mathrm{cm})$. Lizardite is recrystallized into antigorite and subordinate chrysotile, talc, and carbonate. The texture consists of interlocking antigorite flakes or platy crystals with no pseudomorphic replacement. The formation of antigorite in shear planes 
is also characterized by the intense development of talc and chlorite in variably oriented veinlets cutting antigorite crystals (Plate 1, Fig. 3). The veins are themselves deformed by parallel or oblique shear planes. Some of the antigorite is replaced by colorless magnesian chlorite (e.g., Sample 670A$9 \mathrm{R}-1,107-109 \mathrm{~cm}$ ) and by fibrous acicular tremolite. Tremolite fibers are $0.3-0.8 \mathrm{~mm}$ long and grow in the antigorite-rich area of the serpentinites. The tremolite fibers are slightly deformed to undeformed indicating crystallization contemporaneous with the release of strain in the rocks. Tremolite also occur in talc-chlorite-rich sections of the serpentinites, and postdates this assemblage (e.g., Sample 670A-5R-2, 50-52 cm).

Stage 4: Late tensional event serpentinization (e.g., Samples $670 \mathrm{~A}-5 \mathrm{R}-1,111-113 \mathrm{~cm}$, and $670 \mathrm{~A}-9 \mathrm{R}-1,107-109 \mathrm{~cm}$ )

This is a late-stage tensional event that allowed chrysotile to crystallize in newly opened fissures. These veinlets (up to $0.3 \mathrm{~mm}$ thick), though rare (forming about $1 \%$ of the rocks), cut all previously described textures. They represent the latest stage of metamorphism preserved in the Leg 109 serpentinites.

\section{MINERAL CHEMISTRY OF THE METAMORPHIC ASSEMBLAGES}

In this section we present the mineral chemistry of the metamorphic phases and discuss relationships with primary mineral chemistry. The data are discussed in relation to the four stages of serpentinization described above.

\section{Analytical procedures}

$\mathrm{X}$-ray diffraction of the samples was made in order to discriminate serpentine minerals. All thin sections were studied under cathode luminescence in order to detect the presence of $\mathrm{CaCO}_{3}$.

Chemical analyses were determined by electron microprobe analysis using a JEOL model JAX-733 "Superprobe" at Southern Methodist University, Dallas, TX. Normal operating conditions were $15 \mathrm{kV}$ excitation voltage, $20 \mathrm{nA}$ beam current, and $30 \mathrm{~s}$ counting time. Beam diameters were 10 to $20 \mu \mathrm{m}$. Elements analyzed for were sodium, magnesium, aluminum, silicon, sulfur, chlorine, potassium, calcium, titanium, chromium, iron, and nickel. A total of 700 microprobe analyses was performed. A representative set of analyses is reported in Table 1.

Isotope geochemistry analyses were obtained at the University of Wisconsin. Whole-rock oxygen isotope determinations were made on serpentinites using the $\mathrm{BrF}_{5}$ extraction technique and a Finnigan/Mat251 triple collecting mass spectrometer. Isotope ratios are expressed in the staniard per mil notation relative to SMOW (Standard Mean Ocean Water).

\section{Mineral chemistry of the serpentine polymorphs}

Primary and secondary mineral chemistry was compared in order to determine the protolith of the serpentinites. In Figure 3 , we observe that olivine-derived serpentines are the most nickeliferous, followed by orthopyroxene-derived and clinopyroxene-derived pseudomorphs. This pattern of nickel distribution reflects the observed nickel contents of the respective primary silicates (Table 1). Compared to the nickel contents of the primary phases, the nickel contents of serpentinized phases are mostly preserved or increased for pyroxenes, or lowered for olivine. The chromium contents of primary silicates and their corresponding pseudomorphs show the same relationship (Fig. 4). In this case, however, chromium contents are both higher and lower in the pyroxenederived pseudomorphs than recorded in the olivine-derived pseudomorphs reflecting initial chromium values. It is of interest to note that olivine pseudomorphs contain little or no chromium, an observation useful in discriminating this former silicate phase. In Figure 5, the distribution of $\mathrm{Al}_{2} \mathrm{O}_{3}$ between primary silicates and serpentine pseudomorphs is shown.
$\mathrm{Al}_{2} \mathrm{O}_{3}$ appears to behave in a similar manner to $\mathrm{Cr}_{2} \mathrm{O}_{3}$. The olivine-derived pseudomorphs are typically depleted in $\mathrm{Al}_{2} \mathrm{O}_{3}$ making them easily distinguishable from pyroxene pseudomorphs. Unfortunately, the lack of data does not allow us to clearly separate orthopyroxene-derived and clinopyroxene-derived pseudomorphs. However, Figure 5 clearly shows that orthopyroxene-derived pseudomorphs exhibit both gains and losses of $\mathrm{Cr}_{2} \mathrm{O}_{3}$, whereas clinopyroxene-derived pseudomorphs show systematic impoverishment. In Figure 6, which combines the previous aluminumchromium results, we see that the different pseudomorphs cannot be clearly separated on this basis. Dungan (1979) has shown a better defined distribution of orthopyroxene and clinopyroxene pseudomorph chemistry. Our data indicate such a separation, but data on the pyroxene-derived serpentine overlap. FeO content in the orthopyroxene-derived serpentine is generally higher than in the clinopyroxenederived serpentine. We can conclude that serpentine chemistry does partly reflect initial mineral chemistry of primary pyroxene phases. This offers a potential discrimination technique for identifying serpentinite protoliths (e.g., harzburgite vs. Iherzolite). Identification of serpentinite protoliths is of great importance when trying to understand the distribution of refractory and fertile upper mantle material along mid-ocean ridge axes.

\section{OXYGEN ISOTOPE GEOCHEMISTRY}

Whole rock oxygen isotope geochemical results (nine analyses; Table 2) are reported from depths varying from 15 to 85 $\mathrm{m}$ below seafloor in Hole 670A (Fig. 7). Values of $\delta^{18} \mathrm{O}$ (SMOW) range from 3.7 to 8.8 , suggesting final equilibration with hydrothermal fluids that circulated through the peridotites. Seawater is the main constituent of these fluids (Wenner and Taylor, 1973). Temperatures of fluid equilibration with the serpentinites suggested by the $\delta^{18} \mathrm{O}$ data are interpreted to be generally near $200^{\circ} \mathrm{C}$ (see Komor et al., this volume). The results presented here are in the same range of those presented by Wenner and Taylor (1973) and Magaritz and Taylor (1974), and summarized in Moody (1979). Comparison with results from the Sarmiento ophiolite (Elthon et al., 1984) indicates higher temperatures of upper greenschist to actinolite facies $\left(500^{\circ}-600^{\circ} \mathrm{C}\right.$ after Taylor, 1974). It is suggested that Hole $670 \mathrm{~A}$ serpentinites formed in a location where no nearsurface magma source was present. However, in this environment of depressed isotherms, the O-isotopes reflect lower temperatures for a given depth than would be present at normal spreading ridges. Temperatures of the serpentinizing fluids also can be constrained by mineral-fluid equilibria. Maximum temperatures would lie close to the upper stability limit of antigorite, that is, up to $550^{\circ} \mathrm{C}$ at $2 \mathrm{~kb}$, corresponding to low lithostatic pressures (Evans, 1977; Vance and Dungan, 1977) and $\mathrm{XCO}_{2}$ of the fluid up to 0.25 . Increased concentration of aluminum content in lizardite would tend to increase the temperature stability limit to $580^{\circ} \mathrm{C}$ at $2 \mathrm{~kb} \mathrm{PH}_{2} \mathrm{O}$ (Caruso and Chernosky, 1979). Such temperatures involve deep penetration of seawater into oceanic crust $(>5 \mathrm{~km})$ and are consistent with the results of Gregory and Taylor (1981) for the Oman ophiolite $\left(\delta^{18} \mathrm{O}=5.2-13.6\right)$, the results of Elthon et al. (1984) for the Sarmiento ophiolite $\left(\delta^{18} \mathrm{O}=2.6-9.8\right)$; and the results of Spooner et al. (1977) and Heaton and Sheppard (1977) for the Troodos ophiolite $\left(\delta^{18} \mathrm{O}=6.5-7.4\right.$ and 4.4-5.3, respectively).

\section{DISCUSSION}

Peridotites from Hole 670A are variably serpentinized and deformed. Serpentinization products are chiefly dependent upon the protolith composition of the peridotites. Pervasive 
Table 1. Primary and secondary mineral analyses of serpentinized peridotites from Hole 670A.

\begin{tabular}{|c|c|c|c|c|c|c|c|c|c|c|c|c|c|c|c|c|c|}
\hline Sample & Depth $^{1}$ & Ref. & $\mathrm{Na}_{2} \mathrm{O}$ & $\mathrm{MgO}$ & $\mathrm{Al}_{2} \mathrm{O}_{3}$ & $\mathrm{SiO}_{2}$ & $\mathrm{SO}_{3}$ & $\mathrm{Cl}$ & $\mathrm{K}_{2} \mathrm{O}$ & $\mathrm{CaO}$ & $\mathrm{TiO}_{2}$ & $\mathrm{Cr}_{2} \mathrm{O}_{3}$ & $\mathrm{MnO}$ & $\mathrm{FeO}^{*}$ & $\mathrm{NiO}$ & Total & $\operatorname{Min}^{2}$ \\
\hline \multirow[t]{2}{*}{$1 \mathrm{D}-1,19-21 \mathrm{~cm}$} & 6.6 & 475 & 0.02 & 31.61 & 4.88 & 54.74 & 0.01 & 0.0 & 0.01 & 1.23 & 0.0 & 0.89 & 0.10 & 6.01 & 0.09 & 99.58 & opx \\
\hline & & 476 & 0.06 & 31.52 & 5.87 & 37.50 & 0.04 & 0.11 & 0.04 & 0.06 & 0.0 & 0.96 & 0.19 & 6.50 & 0.21 & 83.05 & \\
\hline $3 \mathrm{~W}-1,10-11 \mathrm{~cm}$ & 7.1 & 339 & 0.03 & 31.79 & 5.13 & 53.53 & 0.0 & 0.02 & 0.0 & 2.06 & 0.02 & 0.76 & 0.16 & 6.02 & 0.12 & 99.64 & opx \\
\hline & & 340 & 0.04 & 30.81 & 9.19 & 34.12 & 0.0 & 0.08 & 0.0 & 0.11 & 0.14 & 1.19 & 0.16 & 8.71 & 0.29 & 84.84 & \\
\hline & & 343 & 0.02 & 31.47 & 5.18 & 53.15 & 0.13 & 0.0 & 0.0 & 2.05 & 0.0 & 7.9 & 0.14 & 5.98 & 0.17 & 99.08 & opx \\
\hline & & 345 & 0.03 & 33.18 & 4.84 & 37.78 & 0.0 & 0.24 & 0.03 & 0.04 & 0.0 & 0.38 & 0.16 & 6.92 & 0.42 & 84.01 & \\
\hline $2 \mathrm{D}-1,45-47 \mathrm{~cm}$ & 17.3 & 57 & 0.03 & 32.43 & 5.11 & 55.23 & 0.06 & 0.0 & 0.0 & 1.83 & 0.05 & 0.73 & 0.13 & 5.33 & 0.13 & 101.04 & $\overline{\text { opx }}$ \\
\hline & & 56 & 0.03 & 30.82 & 6.62 & 35.97 & 0.18 & 0.15 & 0.0 & 0.06 & 0.10 & 0.56 & 0.25 & 6.73 & 0.11 & 81.58 & \\
\hline & & 290 & 0.03 & 31.84 & 4.46 & 54.75 & 0.0 & 0.0 & 0.0 & 1.88 & 0.13 & 0.61 & 0.12 & 6.37 & 0.18 & 100.36 & opx \\
\hline & & 291 & 0.07 & 33.02 & 5.35 & 37.09 & 0.16 & 0.07 & 0.02 & 0.05 & 0.05 & 0.79 & 0.26 & 7.42 & 0.20 & 84.54 & \\
\hline & & 293 & 0.03 & 31.05 & 4.86 & 54.33 & 0.02 & 0.0 & 0.0 & 2.55 & 0.04 & 0.77 & 0.16 & 6.37 & 0.16 & 100.36 & opx \\
\hline & & 292 & 0.14 & 35.58 & 2.59 & 42.27 & 0.15 & 0.04 & 0.08 & 0.06 & 0.02 & 0.07 & 0.25 & 3.89 & 0.07 & 85.12 & \\
\hline & & 294 & 0.01 & 32.12 & 4.59 & 55.23 & 0.0 & 0.0 & 0.0 & 1.34 & 0.13 & 0.71 & 0.14 & 5.47 & 0.14 & 100.88 & opx \\
\hline & & 295 & 0.11 & 32.94 & 3.91 & 38.32 & 0.24 & 0.06 & 0.05 & 0.08 & 0.07 & 1.00 & 0.18 & 6.43 & 0.18 & 83.56 & \\
\hline & & 296 & 0.03 & 31.85 & 5.04 & 54.17 & 0.0 & 0.01 & 0.02 & 1.50 & 0.04 & 0.78 & 0.16 & 6.45 & 0.14 & 100.20 & opx \\
\hline & & 297 & 0.03 & 31.82 & 6.42 & 35.56 & 0.30 & 0.12 & 0.02 & 0.03 & 0.03 & 1.27 & 0.29 & 8.23 & 0.19 & 84.30 & \\
\hline & & 298 & 0.0 & 31.11 & 5.32 & 54.17 & 0.0 & 0.0 & 0.01 & 2.00 & 0.09 & 0.85 & 0.14 & 6.52 & 0.16 & 100.36 & opx \\
\hline & & 299 & 0.09 & 31.29 & 7.10 & 34.72 & 0.27 & 0.09 & 0.04 & 0.06 & 0.10 & 1.12 & 0.31 & 8.43 & 0.23 & 83.84 & \\
\hline & & 301 & 0.02 & 31.49 & 4.29 & 54.68 & 0.0 & 0.0 & 0.0 & 2.25 & 0.02 & 0.68 & 0.14 & 6.23 & 0.13 & 99.92 & opx \\
\hline & & 300 & 0.06 & 30.22 & 8.16 & 33.98 & 0.21 & 0.08 & 0.0 & 0.07 & 0.10 & 1.15 & 0.30 & 8.87 & 0.28 & 83.49 & \\
\hline $2 \mathrm{D}-1,54-56 \mathrm{~cm}$ & 17.4 & 214 & 0.03 & 31.32 & 4.64 & 54.59 & 0.0 & 0.01 & 0.01 & 1.23 & 0.05 & 0.76 & 0.13 & 5.68 & 0.07 & 98.52 & opx \\
\hline & & 215 & 0.04 & 32.09 & 6.63 & 36.03 & 0.29 & 0.21 & 0.0 & 0.05 & 0.09 & 1.13 & 0.18 & 5.78 & 0.29 & 82.81 & \\
\hline & & 304 & 0.03 & 31.09 & 4.66 & 53.99 & 0.0 & 0.0 & 0.01 & 2.39 & 0.01 & 0.62 & 0.17 & 6.10 & 0.15 & 99.23 & opx \\
\hline & & 305 & 0.03 & 30.75 & 3.90 & 36.96 & 0.30 & 0.21 & 0.01 & 0.02 & 0.06 & 0.55 & 0.13 & 4.32 & 0.20 & 82.45 & \\
\hline & & 306 & 0.03 & 31.76 & 4.16 & 55.07 & 0.0 & 0.0 & 0.01 & 2.54 & 0.04 & 0.63 & 0.16 & 5.65 & 0.14 & 100.20 & opx \\
\hline & & 307 & 0.02 & 30.25 & 5.59 & 37.06 & 0.09 & 0.09 & 0.0 & 0.03 & 0.05 & 0.99 & 0.07 & 4.77 & 0.20 & 85.20 & \\
\hline & & 312 & 0.02 & 30.05 & 4.69 & 54.29 & 0.04 & 0.0 & 0.0 & 1.60 & 0.08 & 0.68 & 0.16 & 6.19 & 0.11 & 99.91 & opx \\
\hline & & 313 & 0.06 & 29.39 & 9.58 & 34.26 & 0.14 & 0.13 & 0.05 & 0.06 & 0.04 & 1.18 & 0.19 & 8.02 & 0.21 & 83.31 & \\
\hline & & 447 & 0.02 & 32.41 & 4.77 & 54.76 & 0.02 & 0.0 & 0.01 & $\begin{array}{l}.00 \\
1.40\end{array}$ & 0.13 & 0.70 & 0.18 & 6.16 & 0.17 & 100.71 & opx \\
\hline & & 448 & 0.01 & 37.03 & 6.68 & 34.73 & $\begin{array}{l}0.02 \\
0.10\end{array}$ & $\begin{array}{l}0.0 \\
0.21\end{array}$ & 0.02 & 0.04 & 0.04 & 1.50 & 0.08 & 2.86 & 0.19 & 83.05 & \\
\hline & & 308 & 0.16 & 16.74 & 6.25 & 49.83 & 0.0 & 0.0 & 0.0 & 21.04 & 0.09 & 0.99 & 0.12 & 3.18 & 0.07 & 98.48 & $\mathrm{cpx}$ \\
\hline & & 309 & 0.03 & 35.81 & 3.39 & 37.85 & 0.16 & 0.14 & 0.04 & 0.06 & 0.09 & 0.42 & 0.29 & 2.78 & 0.15 & 81.20 & \\
\hline & & 314 & 0.14 & 16.44 & 5.90 & 49.99 & 0.0 & 0.02 & 0.0 & 21.63 & 0.18 & 0.97 & 0.12 & 3.18 & 0.13 & 98.70 & cpx \\
\hline & & 315 & 0.03 & 34.15 & 6.67 & 35.64 & 0.20 & 0.10 & 0.0 & 0.06 & 0.18 & 1.27 & 0.41 & 4.11 & 0.19 & 83.02 & \\
\hline $4 \mathrm{~W}-1,27-30 \mathrm{~cm}$ & 26.6 & 171 & 0.0 & 30.36 & 5.48 & 54.82 & 0.01 & 0.0 & 0.02 & 1.38 & 0.02 & 0.89 & 0.13 & 5.38 & 0.04 & 98.56 & opx \\
\hline & & 172 & 0.04 & 34.46 & 3.51 & 36.41 & 0.26 & 0.36 & 0.0 & 0.46 & 0.12 & 1.01 & 0.27 & 6.19 & 0.12 & 83.20 & \\
\hline & & 176 & 0.04 & 31.78 & 4.02 & 55.71 & 0.0 & 0.02 & 0.0 & 1.11 & 0.10 & 0.51 & 0.16 & 5.73 & 0.17 & 99.36 & opx \\
\hline & & 175 & 0.04 & 33.63 & 1.78 & 37.14 & 0.31 & 0.55 & 0.0 & 0.63 & 0.07 & 0.60 & 0.23 & 6.63 & 0.18 & 81.79 & \\
\hline & & 183 & 0.03 & 31.98 & 3.87 & 56.21 & 0.0 & 0.0 & 0.01 & 1.39 & 0.0 & 0.56 & 0.13 & 5.34 & 0.22 & 99.74 & opx \\
\hline & & 184 & 0.02 & 34.00 & 2.38 & 38.32 & 0.21 & 0.29 & 0.02 & 0.25 & 0.05 & 0.41 & 0.20 & 4.39 & 0.20 & 80.55 & \\
\hline & & 395 & 0.02 & 32.32 & 5.04 & 54.80 & 0.01 & 0.01 & 0.0 & 1.71 & 0.06 & 0.80 & 0.13 & 6.23 & 0.17 & 101.29 & opx \\
\hline & & 396 & 0.05 & 31.80 & 4.72 & 37.85 & 0.09 & 0.14 & 0.0 & 0.82 & 0.11 & 0.62 & 0.15 & 6.09 & 0.11 & 82.55 & \\
\hline & & 397 & 0.03 & 29.98 & 5.01 & 54.61 & 0.01 & 0.0 & 0.01 & 1.91 & 0.03 & 0.82 & 0.11 & 6.31 & 0.14 & 98.96 & opx \\
\hline & & 398 & 0.06 & 34.68 & 3.54 & 37.11 & 0.35 & 0.13 & 0.0 & 0.13 & 0.04 & 0.79 & 0.26 & 5.45 & 0.14 & 82.68 & \\
\hline & & 399 & 0.02 & 32.12 & 5.26 & 54.33 & 0.0 & 0.0 & 0.01 & 1.79 & 0.10 & 0.85 & 0.18 & 6.21 & 0.16 & 101.02 & opx \\
\hline & & 399 & 0.02 & 32.12 & 5.26 & 54.33 & 0.0 & 0.0 & 0.01 & 1.79 & 0.10 & 0.85 & 0.18 & 6.21 & 0.16 & 101.02 & opx \\
\hline & & 400 & 0.04 & 34.66 & 3.17 & 36.47 & 0.30 & 0.32 & 0.01 & 0.89 & 0.07 & 0.63 & 0.23 & 5.05 & 0.15 & 82.00 & \\
\hline & & 401 & 0.03 & 30.92 & 4.71 & 54.54 & 0.05 & 0.01 & 0.01 & 2.06 & 0.11 & 0.77 & 0.19 & 6.22 & 0.17 & 99.79 & opx \\
\hline & & 402 & 0.17 & 31.98 & 4.10 & 41.87 & 0.07 & 0.08 & 0.06 & 2.66 & 0.03 & 0.46 & 0.16 & 5.92 & 0.11 & 87.67 & \\
\hline & & 403 & 0.03 & 32.32 & 4.09 & 54.94 & 0.0 & 0.01 & 0.01 & 2.07 & 0.04 & 0.53 & 0.18 & 6.15 & 0.12 & 100.50 & opx \\
\hline & & 404 & 0.05 & 34.99 & 2.70 & 37.50 & 0.29 & 0.31 & 0.01 & 0.06 & 0.02 & 0.54 & 0.27 & 7.17 & 0.15 & 84.05 & \\
\hline & & 405 & 0.04 & 32.09 & 4.23 & 55.91 & 0.01 & 0.0 & 0.0 & 2.57 & 0.03 & 0.49 & 0.15 & 5.85 & 0.12 & 101.49 & opx \\
\hline & & 406 & 0.04 & 34.12 & 2.43 & 37.90 & 0.36 & 0.23 & 0.01 & 0.56 & 0.04 & 0.46 & 0.22 & 6.73 & 0.12 & 82.31 & \\
\hline & & 421 & 0.0 & 32.95 & 4.15 & 55.52 & 0.0 & 0.0 & 0.0 & 1.72 & 0.06 & 0.53 & 0.17 & 6.22 & 0.11 & 101.44 & opx \\
\hline & & 422 & 0.04 & 34.67 & 2.29 & 35.87 & 0.53 & 0.40 & 0.0 & 0.39 & 0.01 & 0.47 & 0.32 & 6.87 & 0.12 & 81.98 & \\
\hline & & 433 & 0.02 & 32.04 & 4.82 & 54.32 & 0.0 & 0.0 & 0.0 & 1.68 & 0.0 & 0.86 & 0.12 & 6.04 & 0.14 & 100.03 & opx \\
\hline & & 434 & 0.05 & 37.03 & 2.56 & 36.16 & 0.40 & 0.30 & 0.01 & 0.08 & 0.05 & 0.02 & 0.18 & 4.55 & 0.50 & 81.89 & \\
\hline & & 276 & 0.04 & 32.02 & 5.16 & 54.49 & 0.0 & 0.0 & 0.01 & 2.40 & 0.03 & 0.94 & 0.15 & 6.15 & 0.16 & 101.64 & opx \\
\hline & & 275 & 0.04 & 38.49 & 0.03 & 39.32 & 0.11 & 0.13 & 0.0 & 0.04 & 0.01 & 0.0 & 0.08 & 4.90 & 0.38 & 83.54 & \\
\hline & & 417 & 0.16 & 17.07 & 5.77 & 50.91 & 0.01 & 0.02 & 0.0 & 21.41 & 0.11 & 1.00 & 0.16 & 3.21 & 0.10 & 99.92 & cpx \\
\hline & & 418 & 0.04 & 36.63 & 0.50 & 36.63 & 0.31 & 0.56 & 0.0 & 0.11 & 0.03 & 0.03 & 0.16 & 6.34 & 0.54 & 81.88 & \\
\hline & & 423 & 0.20 & 16.91 & 5.11 & 51.74 & 0.0 & 0.01 & 0.0 & 22.48 & 0.15 & 0.92 & 0.13 & 2.69 & 0.08 & 100.41 & $\mathrm{cpx}$ \\
\hline & & 424 & 0.04 & 36.50 & 0.80 & 35.50 & 0.45 & 0.58 & 0.02 & 0.10 & 0.0 & 0.03 & 0.18 & 6.02 & 0.55 & 80.77 & \\
\hline & & $\begin{array}{l}424 \\
425\end{array}$ & 0.16 & $\begin{array}{l}16.18 \\
\end{array}$ & $\begin{array}{l}0.00 \\
4.97\end{array}$ & $\begin{array}{l}35.50 \\
51.45\end{array}$ & $\begin{array}{l}0.43 \\
0.0\end{array}$ & $\begin{array}{l}0.00 \\
0.01\end{array}$ & $\begin{array}{l}0.02 \\
0.0\end{array}$ & $\begin{array}{r}0.10 \\
22.95\end{array}$ & $\begin{array}{l}0.0 \\
0.05\end{array}$ & $\begin{array}{l}0.03 \\
0.82\end{array}$ & $\begin{array}{l}0.10 \\
0.09\end{array}$ & $\begin{array}{l}0.02 \\
2.69\end{array}$ & 0.11 & 99.47 & cpx \\
\hline & & 426 & 0.04 & 36.86 & 0.56 & 36.89 & 0.14 & 0.27 & 0.02 & 0.09 & 0.0 & 0.05 & 0.22 & 6.08 & 0.54 & 81.76 & \\
\hline & & 427 & 0.18 & 16.98 & 5.29 & 51.81 & 0.04 & 0.0 & 0.01 & 22.17 & 0.09 & 0.97 & 0.11 & 3.05 & 0.08 & 100.79 & cpx \\
\hline & & 428 & 0.05 & 36.61 & 1.32 & 36.38 & 0.13 & 0.29 & 0.01 & 0.10 & 0.07 & 0.20 & 0.14 & 6.45 & 0.68 & 82.42 & \\
\hline & & 173 & 0.02 & 47.82 & 0.03 & 41.29 & 0.02 & 0.02 & 0.0 & 0.04 & 0.0 & 0.0 & 0.12 & 8.38 & 0.41 & 98.16 & ol \\
\hline & & 174 & 0.03 & 36.12 & 0.28 & 38.32 & 0.18 & 0.54 & 0.0 & 0.04 & 0.0 & 0.04 & 0.06 & 4.86 & 0.41 & 80.87 & \\
\hline & & 177 & 0.02 & 48.89 & 0.0 & 41.60 & 0.02 & 0.0 & 0.01 & 0.02 & 0.02 & 0.02 & 0.08 & 8.74 & 0.39 & 99.82 & ol \\
\hline & & 178 & 0.03 & 37.41 & 0.0 & 38.70 & 0.19 & 0.35 & 0.0 & 0.04 & 0.0 & 0.0 & 0.12 & 4.86 & 0.32 & 82.02 & \\
\hline & & 179 & 0.01 & 48.95 & 0.0 & 41.48 & 0.04 & 0.0 & 0.0 & 0.04 & 0.02 & 0.0 & 0.13 & 8.58 & 0.40 & 99.64 & ol \\
\hline & & 180 & 0.03 & 38.30 & 0.04 & 31.81 & 0.16 & 1.48 & 0.01 & 0.04 & 0.0 & 0.0 & 0.07 & 7.93 & 0.29 & 80.15 & \\
\hline & & 407 & 0.03 & 50.00 & 0.0 & 40.95 & 0.0 & 0.0 & 0.01 & 0.04 & 0.0 & 0.02 & 0.17 & 9.82 & 0.50 & 101.55 & ol \\
\hline & & 408 & 0.04 & 38.78 & 0.02 & 36.28 & 0.05 & 0.80 & 0.01 & 0.02 & 0.0 & 0.02 & 0.10 & 7.62 & 0.36 & 84.09 & \\
\hline & & 409 & 0.0 & 49.98 & 0.03 & 41.04 & 0.0 & 0.0 & 0.01 & 0.04 & 0.04 & 0.0 & 0.16 & 9.54 & 0.46 & 101.30 & ol \\
\hline & & 410 & 0.03 & 38.28 & 0.04 & 39.12 & 0.19 & 0.16 & 0.0 & 0.05 & 0.0 & 0.02 & 0.07 & 5.21 & 0.43 & 83.59 & \\
\hline & & 411 & 0.01 & $\begin{array}{l}30.20 \\
49.49\end{array}$ & 0.04 & 41.34 & 0.01 & 0.0 & $0.01^{\circ}$ & 0.05 & 0.0 & 0.02 & 0.15 & 9.36 & 0.52 & 101.00 & ol \\
\hline
\end{tabular}


Table 1 (continued).

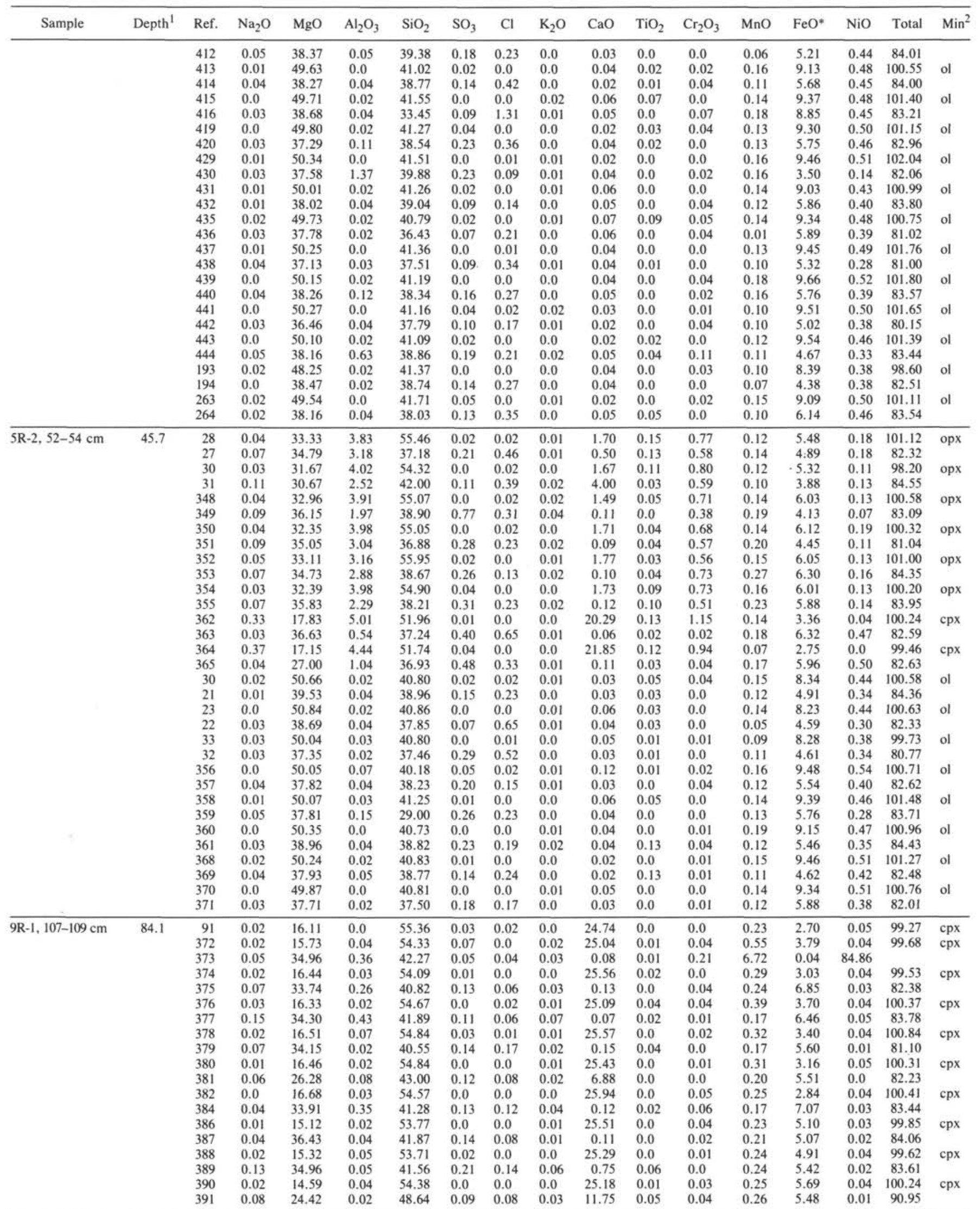

${ }^{1}$ Depth in meters below seafloor. ${ }^{2}$ Analyses divided into analytical pairs - primary mineral and its immediately surrounding secondary phase: opx $=$ orthopyroxene: $\mathrm{cpx}=$ clinopyroxene $; \mathrm{ol}=$ olivine . 


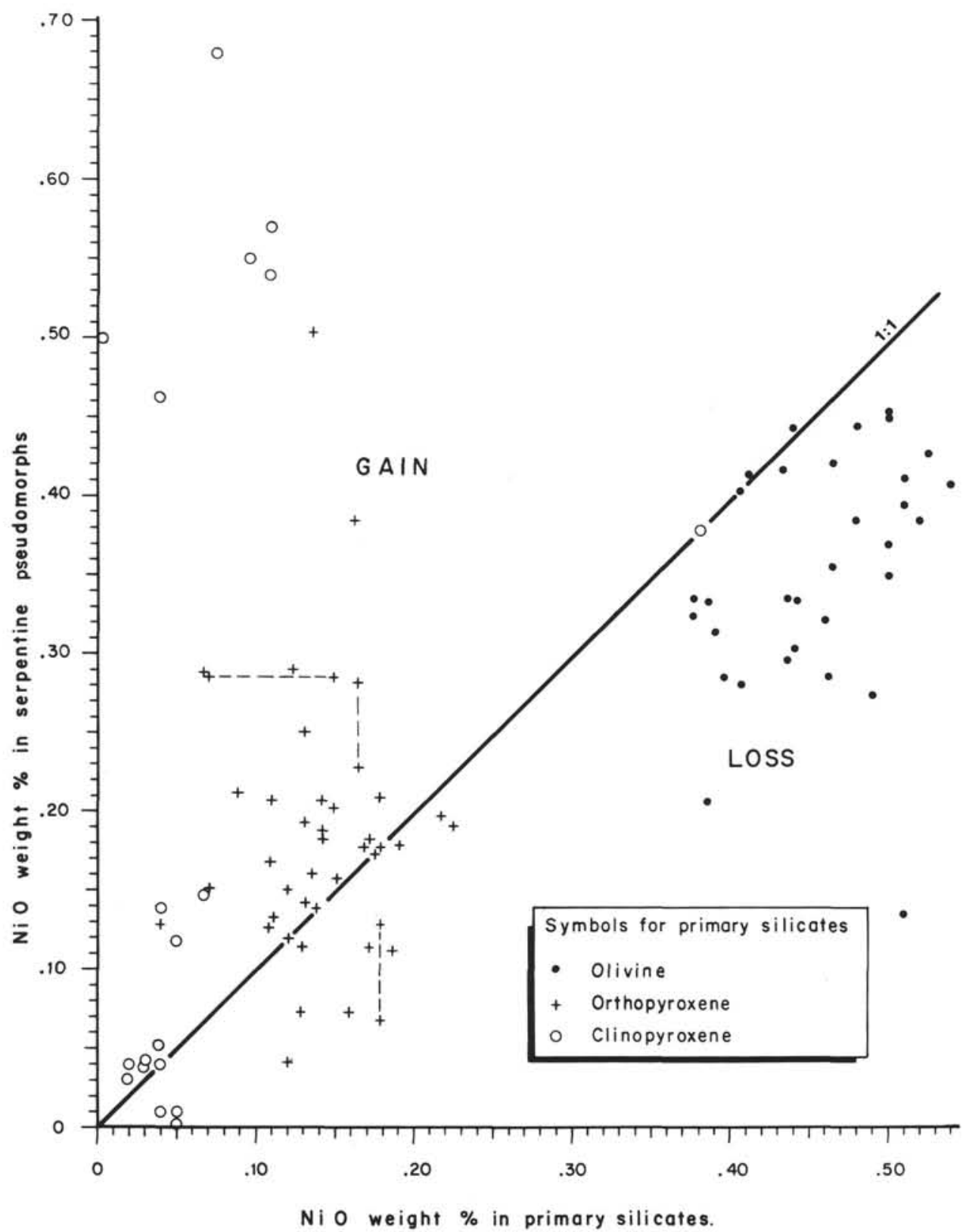

Figure $3 . \mathrm{NiO}$ wt\% partitioning between primary silicates and coexisting lizardite pseudomorphs.

hydration of anhydrous peridotite systems result in the characteristic reactions:

$$
\begin{gathered}
\text { Olivine }+\mathrm{SiO}_{2}+\mathrm{H}_{2} \mathrm{O}=\text { Antigorite }+ \text { Magnetite } \\
\text { Olivine }+ \text { Enstatite }+\mathrm{H}_{2} \mathrm{O}=\text { Serpentine }+ \text { Magnetite }
\end{gathered}
$$

studied respectively by Labotka and Albee (1979) and Bowen and Tuttle (1949). Experimental investigations indicate that when $\mathrm{P}_{\mathrm{t}}=\mathrm{PH}_{2} \mathrm{O}$, the temperatures of these reactions range between $30^{\circ}$ and $500^{\circ} \mathrm{C}$ (see Elthon, 1981, and Janecky and Seyfried, 1986, for reviews). At this stage of very low to low grade metamorphism, lizardite pseudomorphically replaces olivine, and enstatite is stable (Miyashiro et al., 1969; Aumento and Loubat, 1971; Bonatti and Hamlyn, 1981). According to Janecky and Seyfried (1986) reactions (1) and (2) are spontaneous at $300^{\circ} \mathrm{C}(0.5 \mathrm{~kb})$.
Talc formation may be initiated when metamorphic fluid temperatures rise, causing previously formed serpentine to be resorbed during prograde metamorphism (Deer et al., 1962; Caruso and Chernosky, 1979; Moody, 1976, 1979; Elthon, 1981). Talc formation can be accompanied by clinochlore formation:

$$
\begin{gathered}
\text { Lizardite }=\text { Talc }+\underset{(\text { Forsterite }+ \text { Clinochlore }+ \text { Fluid }}{\text { Fo })}
\end{gathered}
$$

or formed directly from enstatite:

$$
\text { Enstatite }+\mathrm{H}_{2} \mathrm{O}=\text { Antigorite }+ \text { Talc }
$$

These two reactions explain the close association of talc with olivine and talc with serpentine (antigorite). Reaction (3) occurs when $\mathrm{PH}_{2} \mathrm{O}=2 \mathrm{~kb}$ and $\mathrm{T}=582^{\circ} \mathrm{C}$ (Caruso and Cher- 


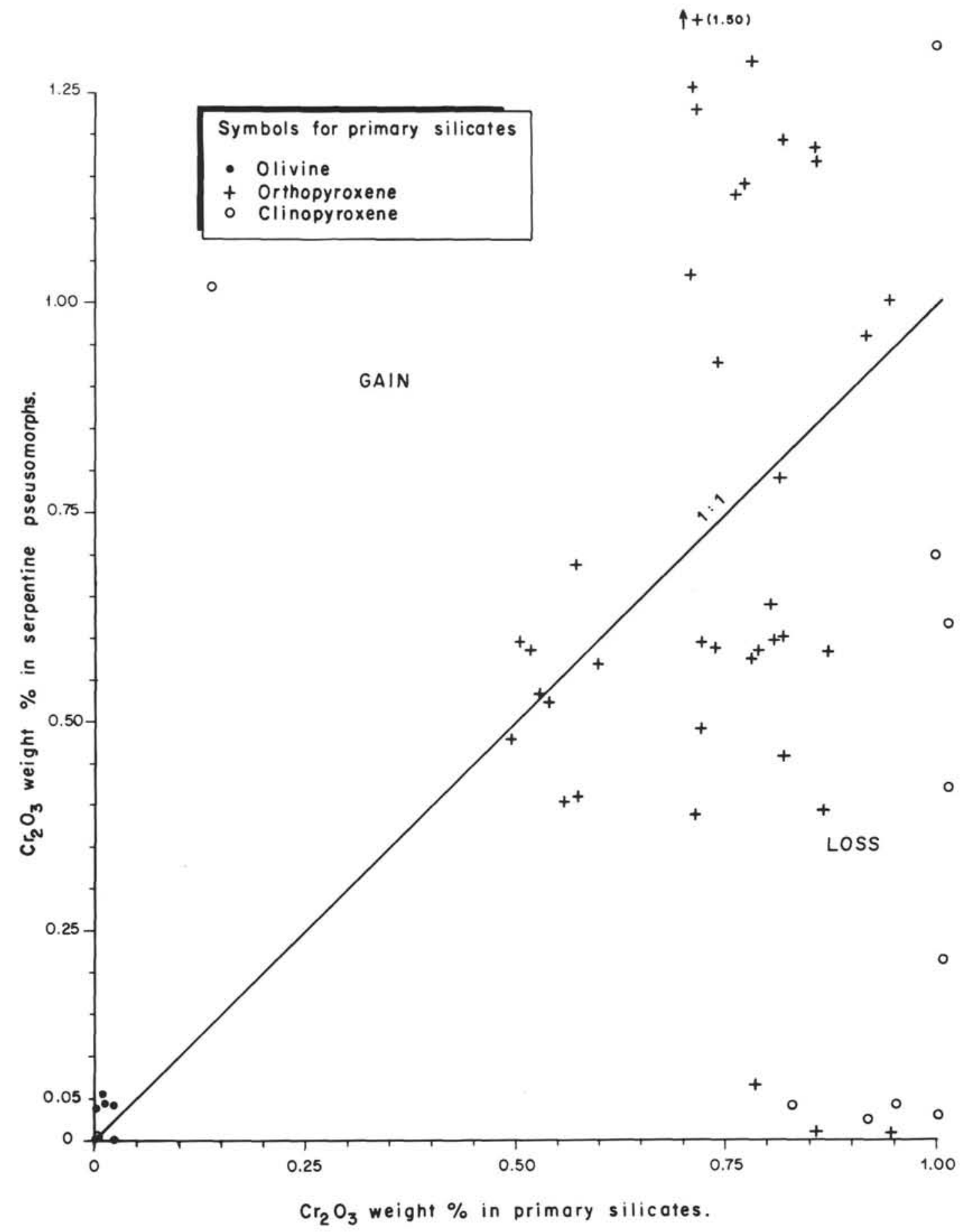

Figure 4. $\mathrm{Cr}_{2} \mathrm{O}_{3}$ wt\% partitioning between primary silicates and lizardite pseudomorphs.

nosky, 1979). However, no olivine more forsteritic than $\mathrm{Fo}_{92}$ was detected in microprobe work suggesting that reaction (4) is the most likely to have occurred. The first appearance of talc also is controlled by the composition of the metamorphic fluid. Recent work by Janecky and Seyfried (1986) has shown that talc formation is favored by high activities of $\mathrm{SiO}_{2}, \mathrm{MgO}$, and $\mathrm{H}^{+}$. The early increase of dissolved $\mathrm{SiO}_{2}$ in metamorphic fluid, caused by hydration of pyroxenes, is likely to lead to talc formation below $300^{\circ} \mathrm{C}$. Consequently, it is very difficult to use talc as a temperature indicator. Evans and Trommsdorff (1970) have shown that talc forms at $310^{\circ} \mathrm{C}$ in high $\mathrm{SiO}_{2}$ fluids, and at $460^{\circ} \mathrm{C}$ if olivine is formed by deserpentinization at $\mathrm{PH}_{2} \mathrm{O}=2 \mathrm{~kb}$. Talc is stable up to $500^{\circ} \mathrm{C}$ for fluids with $\mathrm{XCO}_{2}$ $=0.25$ (Trommsdorff and Evans, 1977). Antigorite locally occurs in small shear zones and forms mainly by replacement of chrysotile (e.g., Sample 670A-5R-1, 111-113 cm). It is in turn replaced by tremolite. Moody (1979) and Elthon (1981) suggested that both high pressure and temperature favor antigorite formation over the other serpentine polymorphs. Antigorite can form in the temperature range of $325^{\circ}-500^{\circ} \mathrm{C}$ and at pressures of less than $3 \mathrm{~kb}$. The antigorite upper stability limit is defined by the reaction: 


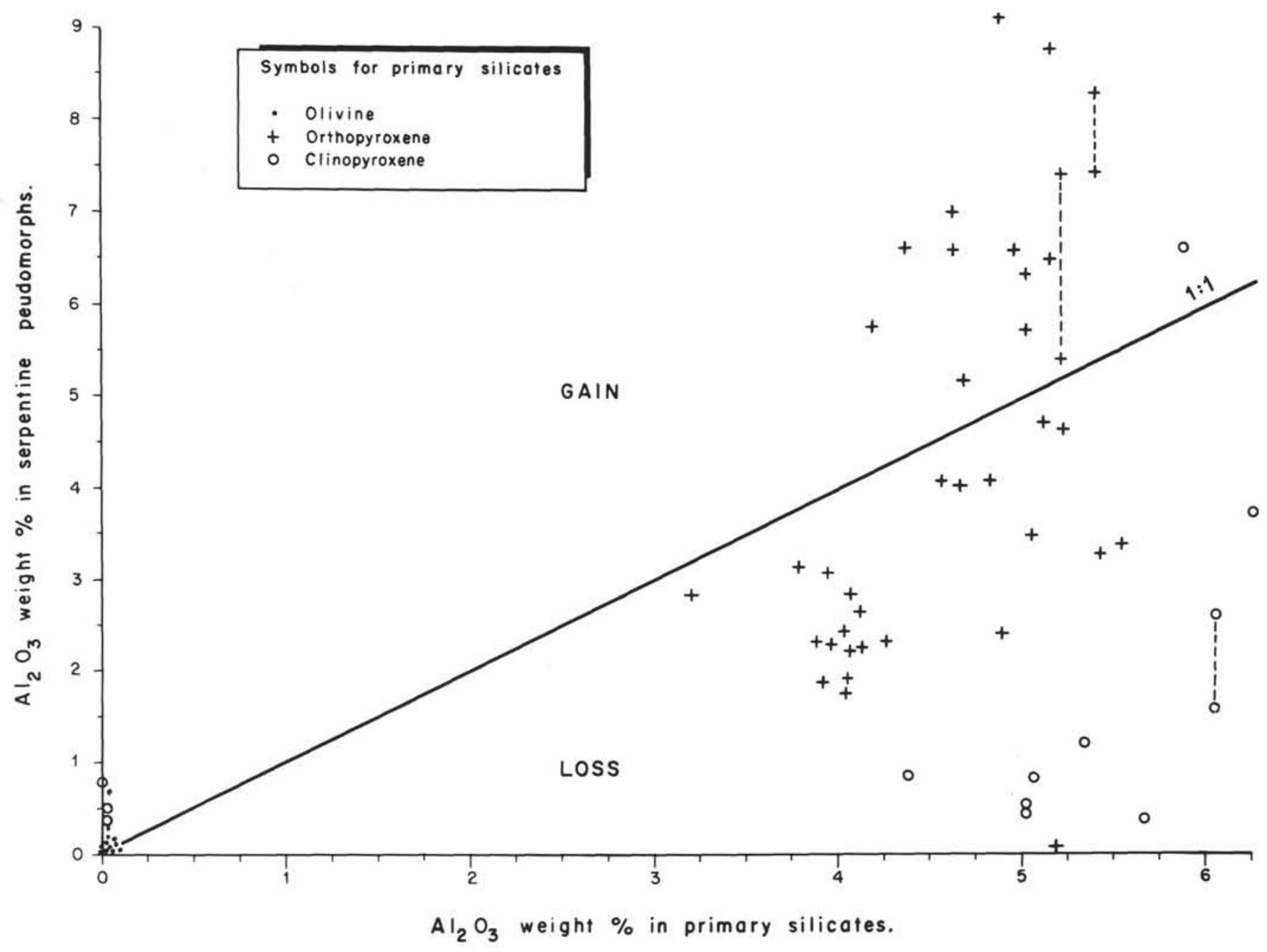

Figure $5 . \mathrm{Al}_{2} \mathrm{O}_{3}$ wt \% partitioning between primary silicates and lizardite pseudomorphs.

$$
\text { Antigorite }+ \text { Diopside }=\text { Tremolite }+ \text { Forsterite }+\mathrm{H}_{2} \mathrm{O}
$$

This reaction is characteristic of medium grade metamorphism of ultramafic rocks (Evans, 1977). Experimental work by Robinson et al. (1982) have shown that reactions involving the formation of talc instead of tremolite occur at $525^{\circ} \mathrm{C}$ and $2 \mathrm{~kb}$.

Clinochlore formation is controlled by reaction (3). The upper stability limit was investigated by Fawcett and Yoder (1966):

$$
\text { Clinochlore }=\text { Forsterite }+ \text { Enstatite }+ \text { Spinel }+\mathrm{H}_{2} \mathrm{O}
$$

and by Seifert (1974):

$$
\begin{gathered}
2 \text { Chlorite }+6 \text { Enstatite }=7 \text { Forsterite }+ \text { Cordierite } \\
+8 \mathrm{H}_{2} \mathrm{O}
\end{gathered}
$$

The temperatures and pressures determined for these reactions are respectively $750^{\circ} \mathrm{C}$ at $3.5 \mathrm{~kb}$ and $700^{\circ} \mathrm{C}$ at $2 \mathrm{~kb}$. Since deserpentinization of olivine was not observed, the upper temperature limit for the metamorphic fluids can be fixed. These temperatures appear to be too high in the particular context of lizardite stability field. Formation of tremolite found in close association with antigorite, chrysotile, talc, and chlorite is favored by calcium-bearing fluids and high water/ rock ratios (Elthon, 1981). The formation of tremolite is controlled by reaction (5) calibrated at $415^{\circ} \mathrm{C}$ and $2 \mathrm{~kb} \mathrm{PH}_{2} \mathrm{O}$ (Evans and Trommsdorff, 1970; Peacock, 1987). Formation of tremolite in the system $\mathrm{MgO}-\mathrm{CaO}-\mathrm{SiO}_{2}-\mathrm{CO}_{2}-\mathrm{H}_{2} \mathrm{O}$ is controlled by $\mathrm{XCO}_{2}$ of the metamorphic fluid. Increasing $\mathrm{XCO}_{2}$ involves an increase in temperature for the first appearance of tremolite, from $\sim 400^{\circ} \mathrm{C}$ for $\mathrm{XCO}_{2}=0$, to $550^{\circ} \mathrm{C}$ for $\mathrm{XCO}_{2}=1.0$ (see review in Mueller and Saxena (1977) and in Peacock (1987)). We conclude that the fluid in Hole $670 \mathrm{~A}$ had a low $\mathrm{XCO}_{2}$. This interpretation agrees with cathode luminescence studies of the Hole $670 \mathrm{~A}$ peridotites where very little carbonate was found. Calcium released during serpentinization of peridotite was partly fixed in the tremolite molecule (Bonatti et al., 1984) and largely reflects the original calcium content of the unserpentinized peridotite (Komor et al., 1985). By combining the various experimental constraints, the likely maximum temperatures of serpentinization and of late metamorphism were in the range of $400^{\circ}$ to $550^{\circ} \mathrm{C}$ at pressures from 2 to $3 \mathrm{~kb}$ with a low $\mathrm{XCO}_{2}$ for the fluid. Further seawater circulation is responsible for actual oxygen isotope ratios and corresponds to a latestage rock-fluid equilibrium (Stakes et al., 1984). This suggests that mineral parageneses can indicate maximum metamorphic temperatures and that oxygen isotopes compositions reflect continued fluid-rock oxygen exchange after the main metamorphic events that are responsible for the secondary mineral assemblages. 


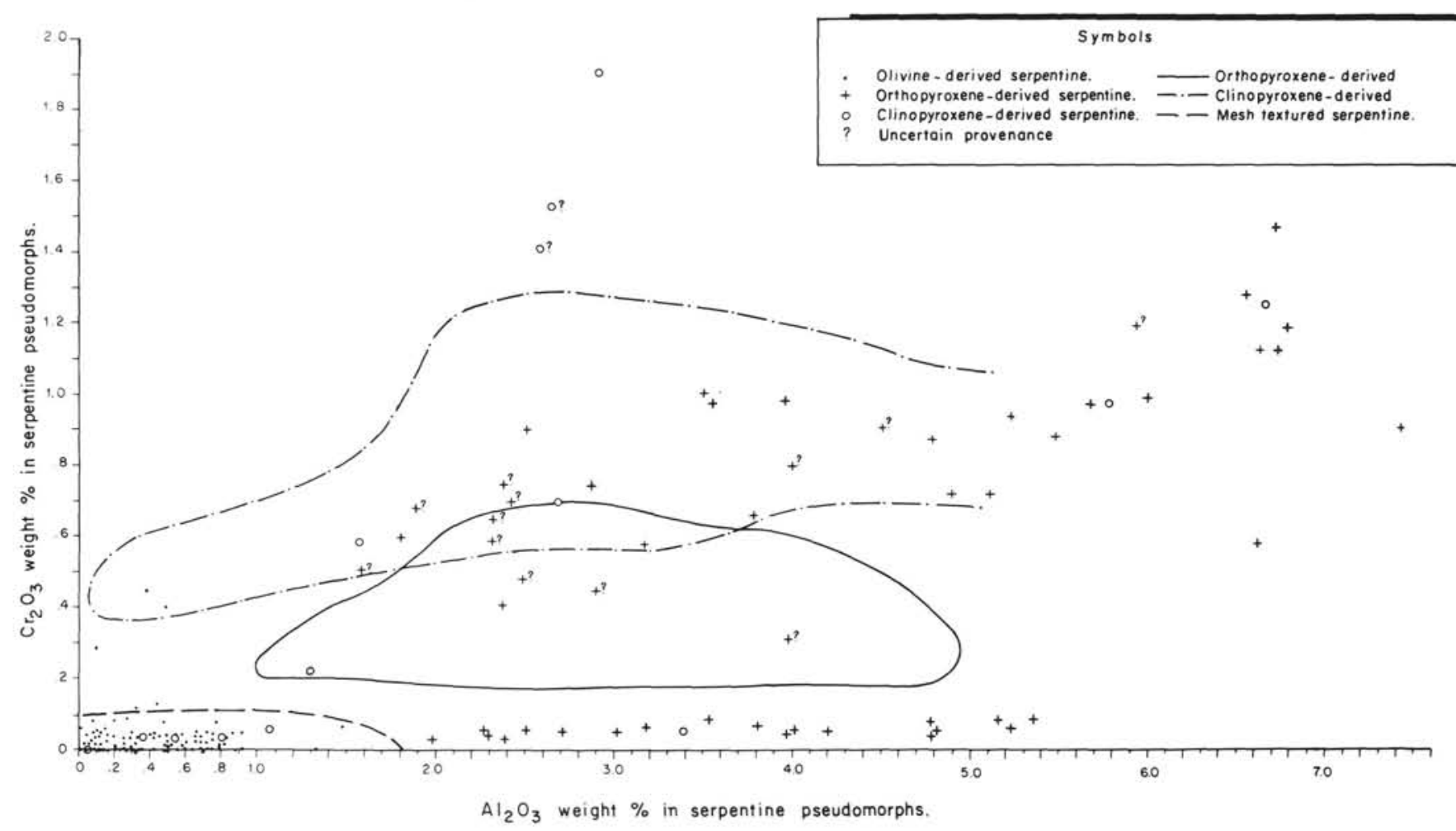

Figure 6. $\mathrm{Cr}_{2} \mathrm{O}$ and $\mathrm{Al}_{2} \mathrm{O}_{3}$ content in wt $\%$ of lizardite pseudomorphs derived from olivine, orthopyroxene, and clinopyroxene.

\section{Origin of Hole 670A serpentinites}

The Hole $670 \mathrm{~A}$ serpentinites result from the pronounced hydration and shearing of refractory upper mantle harzburgites. The pervasive serpentinization provoked a volume increase and a drastic density decrease from 3.3 to $2.6 \mathrm{~g} / \mathrm{cm}^{3}$ (see the "Physical Properties" section in Shipboard Scientific Party, 1988). The hydration of the peridotites was responsible for establishing a sufficiently large gravity gradient to initiate diapiric uprise of the serpentinite body (Bonatti, 1976). There is no indication of how water reached the upper mantle but, it is likely that open fissures, characteristic of slow spreading and non-constructive areas of ridge axes, can provide a path for seawater circulation. In this context, a thin basaltic carapace is a permeable cover and allows virtually unlimited access of seawater to penetrate into upper mantle lying at shallow levels. The diapir would rise along normal faults parallel to the ridge axis. This interpretation is confirmed by observations made of a linear magnetic anomaly on which Hole $670 \mathrm{~A}$ is centered. The diapiric motion is capable of creating dilation fissures, as well as shearing, in response to changing tectonic constraints and volume increases (Francis, 1981; Komor et al., 1985). A diapir model for the emplacement of the Leg 109 serpentinites can also explain the flat foliation observed in recovered cores and submersible dives (Karson, pers. comm., 1986). Similar serpentinized ultramafic intrusions have been described elsewhere (Aumento and Loubat, 1971; Bonatti, 1976) and constitute a significant part of the oceanic crust. This model is also supported by high temperature metamorphic mineral assemblages, which subsequently reequilibrated to lower temperatures, as shown by oxygenisotope results. This progressive reequilibration is likely to occur in a dynamic environment characterized by upward motion, precluding metamorphic equilibrium.
Table 2. Whole-rock oxygen isotope geochemistry of serpentinized periodotites from Hole $670 \mathrm{~A}$ with increasing depth.

\begin{tabular}{llc}
\hline \multicolumn{1}{c}{ Samples } & $\begin{array}{c}\text { Depth } \\
\text { (mbsf) }\end{array}$ & $\delta^{18}$ O-SMOW \\
\hline 2D-1, 20-23 cm & 17 & 8.8 \\
6R-1, 19-22 cm & 54.8 & 7.3 \\
6R-1, 38-40 cm & 55 & 6.2 \\
6R-1, 54-56 cm & 55.1 & 4.7 \\
7R-1, 10-12 cm & 64.1 & 4.3 \\
7R-1, 25-27 cm & 64.3 & 6.8 \\
8R-1, 6-8 cm & 73.6 & 6.7 \\
9R-1, 10-12 cm & 83.1 & 3.7 \\
9R-1, 63-65 cm & 83.6 & 8.1 \\
\hline
\end{tabular}

\section{CONCLUSION}

The Site 670 serpentinized peridotite body is a diapiric intrusion of upper mantle rocks. Petrographic studies show several stages of hydration recording several steps of primary mineral replacement, tectonic constraints, and uplifting. Flat foliation locally disturbed by oblique shearing is related to vertical motion of emplacement. Mineral chemistry of serpentine pseudomorphs and primary silicate phases show important modifications in original $\mathrm{Al}_{2} \mathrm{O}_{3}, \mathrm{NiO}$, and $\mathrm{Cr}_{2} \mathrm{O}_{3}$ contents. However, pyroxene-derived pseudomorphs retain their original $\mathrm{Al}_{2} \mathrm{O}_{3}$ and $\mathrm{Cr}_{2} \mathrm{O}_{3}$ signatures and are easily separated from olivine-derived pseudomorphs. The $\mathrm{FeO}$ content of lizardite pseudomorphs can be used to discriminate between orthopyroxene-derived and clinopyroxene-derived pseudomorphs; $\mathrm{NiO}$ and $\mathrm{Al}_{2} \mathrm{O}_{3}$ contents can also be used for this purpose, but discrimination is more difficult.

Both the metamorphic assemblages and oxygen isotope determinations show that serpentinization of the peridotites 
Leg 109 Serpentinites

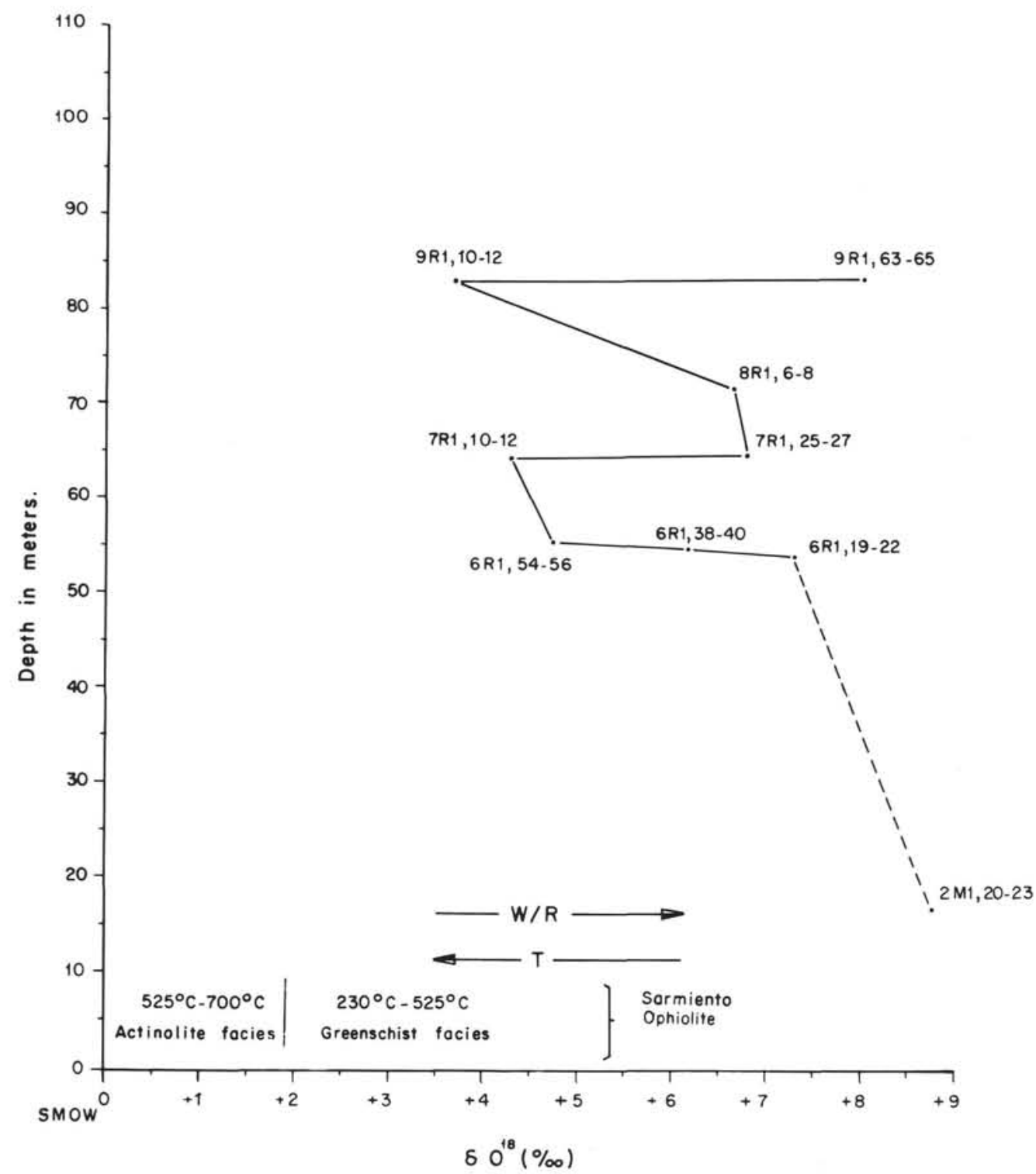

Figure 7. Whole-rock variation of $\delta^{18} \mathrm{O}$ (SMOW) with depth. Values $\delta^{18} \mathrm{O}$ are variable, but plot in the low temperature part of the diagram. Metamorphic facies and indicative temperatures are taken from Elthon (1981) and are based on Taylor (1968) and Apted and Liou (1983) experiments. $\mathrm{T}=$ temperature. W/R $=$ water/rock ratio.

occurred by circulation of heated, water-rich, $\mathrm{CO}_{2}$-poor fluids. Tremolite, talc, clinochlore, and lizardite parageneses suggest metamorphic temperatures ranging from $325^{\circ}$ to $525^{\circ} \mathrm{C}$, at less than $3 \mathrm{~kb}$ total pressure, while oxygen isotope data suggest further reequilibration at temperatures of near $200^{\circ} \mathrm{C}$ for the dominant lizardite-magnetite assemblage. The serpentinization of oceanic upper mantle is favored by thin basaltic crust in non-constructive ridge segments, open fissures related to spreading, and by normal fault planes at rift valley walls.

\section{ACKNOWLEDGMENTS}

This work was granted by the Canadian National Research Council operation grant A1253 to R.Hébert. Funds for oxygen isotope analyses at the University of Wisconsin were provided by the U.S. National Science Foundation (EAR 85-08102) and Gas Research Institute (5086-260-1425). Funds for electron microprobe analyses were provided by the U.S. Science Advisory Council in a grant to
A. C. Adamson. The help of J. W. Valley (University of Wisconsin), Dwight Deuring (Southern Methodist University), Amy Russell (Ocean Drilling Program), Kevin Baker (University of Wisconsin), and of Bill Meyer (Ocean Drilling Program), in the collecting and preparation of the data is gratefully acknowledged.

\section{REFERENCES}

Apted, M. J., and Liou, J. G., 1983. Phase relations among greenschist, epidote-amphibolite, and amphibolite in a basic system. Am. J. Sci., 283A:328-354.

Aumento, F., 1970. Serpentine mineralogy of ultramafic intrusives in Canada and on the Mid-Atlantic Ridge. Pap. Geol. Surv. Can., 69-53:1-51.

Aumento, F., and Loubat, H., 1971. The Mid-Atlantic Ridge near $45^{\circ} \mathrm{N}$, XVI: Serpentinized ultramafic intrusion. Can. J. Earth Sci., 8:631-663.

Bonatti, E., 1976. Serpentine protrusions in the oceanic crust. Earth Planet. Sci. Lett., 32:107-113. 
Bonatti, E., and Hamlyn, P. R., 1981. Oceanic ultramafic rocks. In Emiliani, C. (Ed.), The Sea, The Oceanic lithosphere, v. 7. New York (Wiley), 241-283.

Bonatti, E., Lawrence, J. R., and Morandi, N., 1984. Serpentinization of ocean-floor peridotites. Earth Planet. Sci. Lett., 70:88-94.

Bowen, N. L., and Tuttle, O. F., 1949. The system $\mathrm{MgO}-\mathrm{SiO}_{2}-\mathrm{H}_{2} \mathrm{O}$. Geol. Soc. Am. Bull., 60:439-460.

Caruso, L. J., and Chernosky, J. V., Jr, 1979. The stability of lizardite. Can. Mineral., 17:757-769.

Detrick, R. S., Fox, P. J., Schulz, N., Pockalny, R., Kong, L., Mayer, L., and Ryan, W.B.F., 1988. Geologic and tectonic setting of the MARK area. In Detrick, R., Honnorez, J., Bryan, W. B., Juteau, T., et al., Proc. ODP, Init. Repts., 106/109: College Station, TX (Ocean Drilling Program), 15-22.

Dungan, M. A., 1979. A microprobe study of antigorite and some serpentine pseudomorphs. Can. Mineral., 17:771-784.

Deer, W. A., Howie, R. A., and Zussman, J., 1962. An introduction to rock-forming minerals, Vol. 3, Sheet Silicates. London (Longman), $528 \mathrm{p}$.

Elthon, D., 1981. Metamorphism in oceanic spreading centers. In Emiliani, C. (Ed.), The Sea, The Oceanic Lithosphere, v. 7. New York (Wiley), 285-303.

Elthon, D., Lawrence, J. R., Hanson, R. E., and Stern, C., 1984. Modelling of oxygen-isotope data from Sarmiento ophiolite complex, Chile. In Gass, I. G., Lippard, S. J., and Shelton, A. W. (Eds.), Ophiolites and oceanic lithosphere. Oxford (Blackwell Sci. Publ.), 185-197.

Evans, B. W., 1977. Metamorphism of alpine peridotite and serpentinite. Ann. Rev. Earth Planet. Sci. Lett., 5:397-447.

Evans, B. W., and Trommsdorff, V., 1970. Regional metamorphism of ultramafic rocks in the Central Alps. Parageneses in the system $\mathrm{CaO}-\mathrm{MgO}-\mathrm{SiO}_{2}-\mathrm{H}_{2} \mathrm{O}$. Schweiz. Min. Pet. Mitt., 50:481-492.

Fawcett, J. J., and Yoder, H. S., 1966. Phase relationships of chlorites in the system $\mathrm{MgO}-\mathrm{Al}_{2} \mathrm{O}_{3}-\mathrm{SiO}_{2}-\mathrm{H}_{2} \mathrm{O}$. Am. J. Sci., 51:353-380.

Ferry, J. M., Mutti, L. J., and Zuccala, G. J., 1987. Contact metamorphism, hydrothermal alteration of Tertiary basalts from the Isle of Skye, northwest Scotland. Contrib. Mineral. Petrol. 95:166-181

Francis, T.J.G., 1981. Serpentine faults, J. Geophys, Res., $86: 11616-11622$

Gregory, R. T., and Taylor, H. P., Jr., 1981. An oxygen profile in section of Cretaceous ocean crust, Semail Ophiolite, Oman: Evidence for $\delta^{18} \mathrm{O}$ buffering of the oceans by deep $(>5 \mathrm{~km})$ seawater-hydrothermal circulation of mid-ocean ridges. J. Geophys. Res., 86:2737-2755.

Heaton, T.H.E., and Sheppard, S.M.F., 1977. Hydrogen and oxygen isotope evidence for seawater hydrothermal alteration and ore deposition, Troodos Complex. In Gass, I. G. (Ed.), Volcanic Processes in Ore Genesis, Geol. Soc. London Spec. Publ., 7:42-57.

Janecky, D. R., and Seyfried, W. E., Jr., 1986. Hydrothermal serpentinization of peridotite within the oceanic crust: Experimental investigations of mineralogy and major element chemistry. Geochim. Cosmochim. Acta, 50:1357-1378.

Komor, S. C., Elthon, D., and Casey, J. F., 1985. Serpentinization of cumulate ultramafic rocks from the North Arm Mountain massif of the Bay of Islands ophiolite. Geochim. Cosmochim. Acta, 49:2331-2338.

Labotka, T. C., and Albee, A. L., 1979. Serpentinization of Belvedere Mountain ultramafic body, Vermont: Mass balance and reaction at the metasomatic front. Can. Mineral., 17:831-845.
Macdonald, A. H., and Fyfe, W. S., 1985. Rate of serpentinization of ultramafic rocks in oceanic environments and continental ophiolite complexes. Tectonophysics, 116:123-135.

Magaritz, M., and Taylor, P. H., 1974. Oxygen and hydrogen isotope studies of serpentinization in the Troodos ophiolite complex. Earth Planet. Sci. Lett., 23:8-14.

Miyashiro, A., Shido, F., and Ewing, M., 1969. Composition and origin of serpentinites from the Mid-Atlantic Ridge near $24^{\circ}$ and $20^{\circ}$ North latitude. Contrib. Mineral. Petrol., 23:117-127.

Moody, J. B., 1976. Serpentinization. A review. Lithos, 9:125-138. 1979. Serpentines, spilites and ophiolite metamorphism. Can. Mineral., 17:871-887.

Mueller, R. F., and Saxena, S. K., 1977. Chemical Petrology, New York (Springer-Verlag), 235-239.

Peacock, S. M., 1987. Serpentinization and infiltration metasomatism in the Trinity peridotite, Klamath province, northern California: Implications for subduction zones. Contrib. Mineral. Petrol., 95:55-70.

Riordon, P. H., 1955. The genesis of asbestos in ultrabasic rocks. Econ. Geol., 50:50-65.

Robinson, G. R., Jr., Hoas, J. L., Jr., Schafer, C. M., and Haselton, H. T., Jr., 1982. Thermodynamic and thermophysical properties of selected phases in the $\mathrm{MgO}-\mathrm{SiO}_{2}-\mathrm{H}_{2} \mathrm{O}-\mathrm{CO}_{2}, \mathrm{CaO}-\mathrm{Al}_{2} \mathrm{O}_{3}-\mathrm{SiO}_{2}$ $\mathrm{H}_{2} \mathrm{O}-\mathrm{CO}_{2}$, and $\mathrm{Fe}-\mathrm{FeO}_{2}-\mathrm{Fe}_{2} \mathrm{O}_{3}-\mathrm{SiO}_{2}$ chemical systems with special emphasis on the properties of basalts and their mineral components. U.S. Geol. Surv. Open-file Rept., 83-79, 429 p.

Seifert, G. B., 1974. An experimental model for low pressure metamorphism of siliceous dolomitic marble. Am. J. Sci., 274:487-509.

Shipboard Scientific Party, 1988. Site 670. In Bryan, W. B., Juteau, T., et al., Proc. ODP, Init. Repts., 106/109: College Station, TX (Ocean Drilling Program), 203-237.

Spooner, E.T.C., Chapman, H. S., and Smewing, J. D., 1977. Strontium isotopic contamination and oxidation during ocean floor hydrothermal metamorphism of the ophiolitic rocks of the Troodos massif, Cyprus. Geochim. Cosmochim. Acta, 41:873-890.

Stakes, D. S., Taylor, H. P., Jr., and Fisher, R. L., 1984. Oxygenisotope and geochemical characterization of hydrothermal alteration in ophiolite complexes and modern oceanic crust. In Gass, 1. C., Lippard, S. J., and Shelton, A. W. (Eds.), Ophiolites and Oceanic Lithosphere. Oxford (Blackwell Sci. Publ.), 199-214.

Taylor, H. P., Jr., 1968. The oxygen isotope geochemistry of igneous rocks. Contrib. Mineral. Petrol., 19:1-71.

1974. The application of oxygen and hydrogen isotope studies to problems of hydrothermal alteration and ore deposition. Econ. Geol., 69:843-883.

Trommsdorff, V., and Evans, B. W., 1977. Antigorite-ophicarbonates. Phase relations in a portion of the system $\mathrm{CaO}-\mathrm{MgO}$ $\mathrm{SiO}_{2}-\mathrm{H}_{2} \mathrm{O}-\mathrm{CO}_{2}$. Contrib. Mineral. Petrol., 60:39-56.

Vance, J. A., and Dungan, M. A., 1977. Formation of peridotites by deserpentinization in the Darrington and Sultan areas Cascade Mountains, Washington. Geol. Soc. Am. Bull., 88:1497-1508.

Wenner, D. B., and Taylor, H. P., Jr., 1973. Oxygen and hydrogen isotope studies of the serpentinization of ultramafic rocks in oceanic environments and continental ophiolite complexes. Am.J. Sci., 273:207-239.

Date of initial receipt: 17 October 1988

Date of acceptance: 30 May 1989

Ms 106/109B-129 


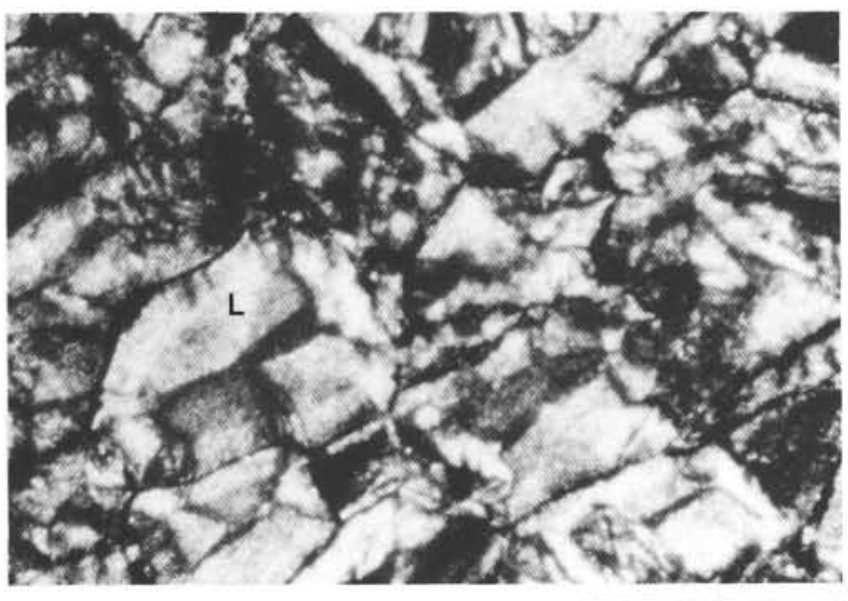

1

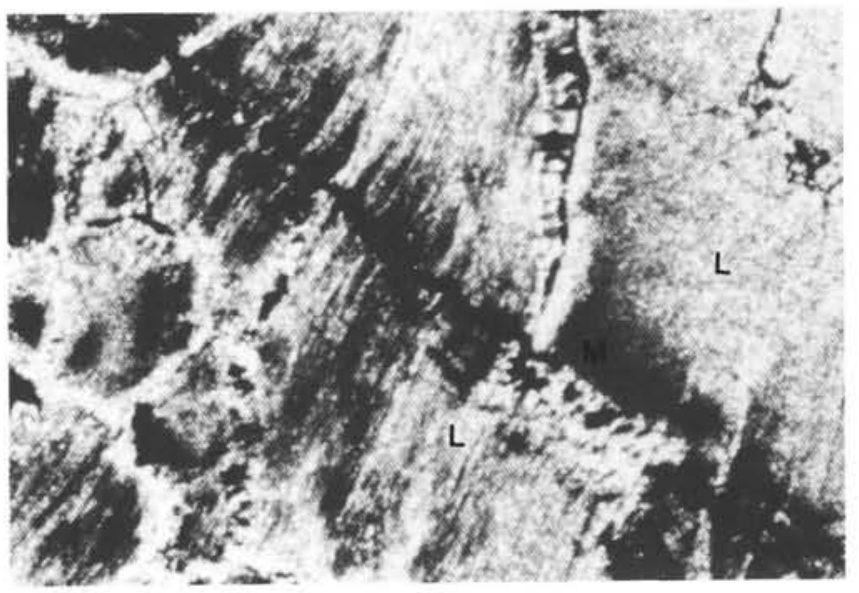

2

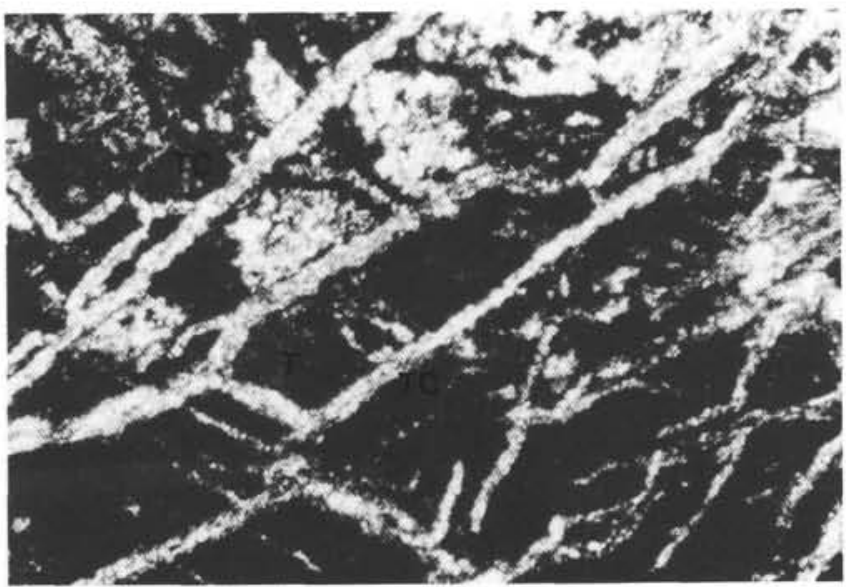

3

Plate 1. 1. Well-developed mesh texture of lizardite (L) after olivine. Thin section from Sample 109-670A-9R-1, 10-12 cm. Scale bar is $1 \mathrm{~mm}$ long. 2. Serrate texture: magnetite (M) infiltration from a magnetite veinlet into lizardite (L). Thin section from Sample 109-670A-3W-I, 12-14 cm. Scale bar is $0.5 \mathrm{~mm}$ long. 3. Crosscutting relationships: talc $(\mathrm{T})$ veinlet is displaced by younger talc $(\mathrm{T})+$ chlorite $(C)$ veinlet. Thin section from Sample 109-670A-5R-2, 50-52 cm. Scale bar is $1 \mathrm{~mm}$ long. 